\title{
Solution of Fuzzy Initial Value Problems Using Homotopy Analysis Method and Padè Approximate
}

\author{
Rana H. Ali' ${ }^{1}$ Kais I. Ibraheem² \\ ${ }^{1}$ Department of Mathematics, College of Education for Pure Science, University of Mosul, Mosul, Iraq \\ ${ }^{2}$ Department of Computer, College of Education for Pure Science, University of Mosul, Mosul, Iraq \\ Email: rana.esp128@student.uomosul.edu.iq, kaisiamail@yahoo.com
}

How to cite this paper: Ali, R.H. and Ibraheem, K.I. (2020) Solution of Fuzzy Initial Value Problems Using Homotopy Analysis Method and Padè Approximate. Open Access Library Journal, 7: e6803. https://doi.org/10.4236/oalib.1106803

Received: September 8, 2020

Accepted: October 12, 2020

Published: October 15, 2020

Copyright $\odot 2020$ by author(s) and Open Access Library Inc.

This work is licensed under the Creative Commons Attribution International License (CC BY 4.0).

http://creativecommons.org/licenses/by/4.0/

(c) (i) Open Access

\begin{abstract}
In this paper, we have studied and developed some analytical methods for solving some linear and fuzzy differential equations of the first degree with their fuzzy elementary conditions using the homotopy analytical method, using the approximate Padè method, and comparing the results with the exact solution of the equation, which indicates that the error decreases exponentially with the $\mathrm{N}$ degree of approximation. Also, we made some improvements to the solution results by using Padè enhancements as shown in the two examples with tables and figures.
\end{abstract}

\section{Subject Areas}

Numerical Analyses

\section{Keywords}

Homotopy Analytical Method, Fuzzy Original Value Problems, Padè Approximation

\section{Introduction}

In this thesis, we solve the equation linear through a systematic method called the homotopy trepidation method (HPM). The Chinese mathematician He first proposed this method in 1999 [1], the mysterious logic (fuzzy). It provides the general framework for solving the problem of information representation approximate or completely non-specific and provides the mechanism needed to use this information. The ambiguous (fuzzy) logic focuses on the conclusion through non-specific expressions and vocalizations such as young, long, short, 
and these terms are called linguistic or fuzzy variables. Linear fuzzy differential equations play an essential role in modeling science and engineering problems, and there are many ways to find a solution to them. Lotfi-Zadeh (1965) proposed a complex logic that Professor Zadeh discussed fuzzy set theory, fuzzy number concept, and arithmetical operation with these numbers in [2] [3] and following there are many articles and books published about fuzzy systems, Fuzzy set theory is considered an effective way for modeling and ambiguous or self-processing information in mathematical models. Their vital trends of expansion have been varied and it is applicable to the diverse real problems, for example, in the golden mean [4], systems of fractional differential equations [5], engineering problems [6], models [7], particle systems [8], medicine [9], non-linear water waves [10], civil engineering [11], Klein-Gordon equation [12], discontinuity with solitary waves [13], physics [14], and in modeling hydraulic [15], unsteady boundary-layer flows [16], boundary value problems for integro-differential equations [17], MHD fluid flow and heat transfer problem [18], fractional SIR model [19], the researcher in [20] explained (FIVPs) as a family of differential inclusions. The homotopy method is not dependent on small parameters, the homotopy method was used with Padè approximation [21], the iterative heterogeneous method; the (HAM) which is introduced by Liao [22], is beneficially and conveniently used to resolution certain types of linear and non-linear problems, one of the advantages of the (HPM) is that (HPM) solution series is only dependent upon two factors. There is no problem with this sentence and it has been validated. The main advantage of HPM is the flexibility to give approximate and exact solutions to both linear and non-linear problems. Approximation solution for fuzzy initial value problems of ordinary differential equations then it is:

$$
x^{\prime}(t)=f(t, x(t)), t_{0} \leq t \leq t_{0}+a
$$

Subject to the initial conditions

$$
x\left(t_{0}\right)=x^{0}
$$

In this paper, we used the homotopy, analytical, blurry method with Padè Approximants to solve elementary values' problems.

\section{Definitions}

In this paper, show some basic definitions.

Definition of Fuzzy number 2.1 [23] It is a fuzzy subdivision of with normal, curving, and an upper semi-continuous relationship function of partial support for each $r \in[0,1]$ it is called fuzzy number and the set

$$
[u]^{r}=\{s \in R: u(s) \geq r\}
$$

And also

$$
[u]^{0}=\{s \in R: u(s)>0\}
$$


It is easy to prove that $u$ is a fuzzy number if and only if $[u]^{r}$ is a convex compressed a subset of $R$ for each $r \in[0,1]$ and $[u] \neq \varnothing$ and so on if $u$ is a fuzzy number then

$$
\begin{gathered}
{[u]^{r}=[\underline{u}(r), \bar{u}(r)]} \\
\underline{u}(r)=\min \left\{s: s \in[u]^{r}\right\} \\
\bar{u}(r)=\max \left\{s: s \in[u]^{r}\right\}
\end{gathered}
$$

For each $r \in[0,1],[u]^{r}$ is called an $r$-cut representation or a parameter form of a fuzzy number.

$u: R \rightarrow[0,1]$ defined by

$$
u(s)=\sup \{r: \underline{u}(r) \leq s \leq \bar{u}(r)\}
$$

Theorem 2.2 [24] If we have the two fuzzy numbers $U$ and $V$, so for each $r \in[0,1]$, we have

(i) $[u+v]^{r}=[u]^{r}+[v]^{r}=\left[\underline{u}_{r}+\underline{v}_{r}, \bar{u}_{r}+\bar{v}_{r}\right]$

(ii) $[\lambda u]^{r}=\lambda[u]^{r}= \begin{cases}{[\lambda \bar{u} r, \lambda \underline{u r}]:} & \lambda \geq 0 \\ {[\lambda \bar{u} r, \lambda \underline{u} r]:} & \lambda<0\end{cases}$

(iii) $[u v]^{r}=[u]^{r}[v]^{r}=\left[\min S_{r}, \max S_{r}\right]$, where

$$
S_{r}=\left\{\underline{u}_{r} \underline{v}_{r}, \underline{u}_{r} \bar{v}_{r}, \bar{u}_{r} \underline{v}_{r}, \bar{u}_{r} \bar{v}_{r}\right\}
$$

All the fuzzy numbers that are collected by adding and multiplying are defined by part (i) and (ii), is a convex cone [25].

Definition 2.3 [26] Let us have the comprehensive set $E=\{a, b, c, d, e\}$. That $A$ is a subset of $\mathrm{E}$ ( $A \subseteq E$ ) for each of these elements has one possibility of two probabilities, and it is an element of $A$ or not, so the function the characteristic is 0 or 1. If the membership function was not zero or one, but its value is anything from zero and one, and thus we notice the membership function is located between zero and one, meaning the closed period $[0,1]$, it's called fuzzy set we can write as follows:

1) If $\mu_{A}(X)=0$ if it is not an element in $A$.

2) If $\mu_{A}(X) \simeq 1$ if the degree of belonging is high of $A$.

3) If $\mu_{A}(X) \simeq 0$ if the degree of belonging is weak.

4) If $\mu_{A}(X)=1$ if zero is an element in $A$.

$$
\begin{gathered}
\mu_{A}(X): E \rightarrow[0,1] \\
\mu_{A}(X)= \begin{cases}1 & x \in A \\
0 & x \notin A\end{cases}
\end{gathered}
$$

Definition 2.4 [27] Padè approximation is a well-known approximation of functions. This approximation dates back to the French scientist Henri Eugene Padè (1863-1953). This approximation approximates the function and turns it into a relative function, so we take the polynomial function $f(x)$ in the period $[a, b]$, and we take the Padè approximations for this function. The Padè ap- 
proximations try to reduce the value of the most significant error in the period $[a, b]$ any part of this period; we get the division of polynomials $Q_{M}(x)$ by $P_{N}(x)$ of degree $N$ and $M$ respectively. It is symbolized by the symbol $[N / M]$ or $P A[N / M]$ since $N$ and $M$ are normal numbers, we will symbolize this division with the symbol $R_{N, M}(x)$ as follows:

$$
R_{N, M}(x)=P A[N / M]=\frac{P_{N}(x)}{Q_{M}(x)}, a \leq x \leq b
$$

$Q_{M}(x), P_{N}(x)$ Polynomials are solved so that the function $f(x)$ and $R_{N, M}(x)$ plus their derivatives to the $N+M$ rank are identical when $x=0$, Assuming that $f(x)$ is analytical and has a (Maclaurin Series), we get:

$$
f(x)=a_{0}+a_{1} x+a_{2} x^{2}+\cdots+a_{k} x^{k}+\cdots
$$

\section{Homotopic Analytical Method (HAM) [28]}

I will discuss some of basic concepts of the homotopy method of analysis.

If we have the following non-linear equation

$$
N[y(t)]=0, t \geq 0
$$

Since $N$ represents a non-linear operator, $y$ is an unknown function, and $t$ is an independent variable. The zero-order distortion equation was derived by generalizing and formulating the homotopy method:

$$
(1-q) L\left[\varnothing(t, q)-y_{0}(t)\right]=q \bar{h} H(t) N[\varnothing(t, q)]
$$

Since $q \in[0,1]$ the inclusion parameter is called the homotopy parameter, $L$ is the auxiliary linear bound that achieves the property of $L(0)=0$ when $L(y)=0, \varnothing(t, q)$ solve Equation (15), $y_{0}(t)$ initial estimation of the exact solution $y(t), \bar{h} \neq 0$ is called the convergence control parameter, $H(t) \neq 0$ auxiliary function, respectively, within the homotopy we can have great freedom to choose the auxiliary linear term $L$, the initial estimate $y_{0}(t)$, And the parameters $\bar{h}, H(t)$ when $q=0$, due to the property $L(0)=0$, we get from Equation (16) the solution:

$$
\varnothing(t, 0)=y_{0}(t)
$$

When $q=1$, and since $H(t) \neq 0, \bar{h} \neq 0$, and Equation (15) is equivalent to Equation (16) then:

$$
\varnothing(t, 1)=y(t)
$$

As $y(t)$ is a solution to the original Equation (15) and so whenever the homotopy parameter $q$ increases from zero to one, the solution $\varnothing(t, q)$ changes continuously from the initial guess $y_{0}(t)$ to the exact solution $y(t)$, this A type of constant change in solution is called homotypic deformation. By expanding $\varnothing(t, q)$ using the Taylor series with respect to $q$, we get the following:

$$
\varnothing(t, q)=y_{0}(t)+\sum_{m=1}^{+\infty} y_{m}(t) q^{m}
$$




$$
y_{m}(t)=\left.\frac{1}{m !} \frac{\partial^{m} \varnothing(t, q)}{\partial q^{m}}\right|_{q=0}
$$

If $h, H(t), y_{0}(t), L$ are correctly chosen then Equation (19) approaches when $q=1$, therefore based on this hypothesis, the solution becomes as follows:

$$
y(t)=\varnothing(t, 1)=y_{0}(t)+\sum_{m=1}^{+\infty} y_{m}(t)
$$

Now we know the vector $\boldsymbol{y}_{n}(t)$ as follows:

$$
\boldsymbol{y}_{n}(t)=\left\{y_{0}(t), y_{1}(t), y_{2}(t), \cdots, y_{n}(t)\right\}
$$

By deriving the zero deformation Equation (16), $m$ times for parameter $q$, then substituting $q$ for zero, and finally, we divide the equation by $m$ ! (the deformation equations) of the order $\mathrm{m}$ are obtained as follows:

$$
L\left[y_{m}(t)-x_{m} y_{m-1}(t)\right]=\bar{h} H(t) R_{m-1}\left(\boldsymbol{y}_{m-1}(t)\right)
$$

According to the conditions

$$
y_{m}(0)=0
$$

As that

$$
\begin{gathered}
R_{m-1}\left(\boldsymbol{y}_{m-1}(t)\right)=\left.\frac{1}{(m-1) !} \frac{\partial^{m-1} N[\varnothing(t, q)]}{\partial q^{m-1}}\right|_{q=0} \\
x_{m}= \begin{cases}0 & m \leq 1 \\
1 & m>1\end{cases}
\end{gathered}
$$

\section{An Algorithm for Solving Fuzzy Initial Values Problems Using the Homo-Analytical Method with Padè Approximations}

We will demonstrate the fuzzy homotopy analytic method with Padè approximants to solve elementary value problems:

Case I. If $x(t)$ is differentiable,

$$
\left[D_{1} x(t)\right]^{r}=\left[\underline{x}_{r}^{\prime}(t), \bar{x}_{r}^{\prime}(t)\right]
$$

To solve (FIVP) we follow the following steps:

Step (i): to solve the system of (ODEs) subsequent for $\underline{x}_{r}(t), \bar{x}_{r}(t)$ :

$$
\begin{aligned}
& \underline{x}_{r}^{\prime}(t)=f_{1, r}\left(t, \underline{x}_{r}(t), \bar{x}_{r}(t)\right) \\
& \bar{x}_{r}^{\prime}(t)=f_{2, r}\left(t, \underline{x}_{r}(t), \bar{x}_{r}(t)\right)
\end{aligned}
$$

Subject to the initial conditions

$$
\begin{aligned}
& \underline{x}_{r}\left(t_{0}\right)=\underline{x}_{r}^{0} \\
& \bar{x}_{r}\left(t_{0}\right)=\bar{x}_{r}^{0}
\end{aligned}
$$

Step (ii): ensure that the solution $\left[\underline{x}_{r}(t), \bar{x}_{r}(t)\right]$ and its derivative $\left[\underline{x}_{r}^{\prime}(t), \bar{x}_{r}^{\prime}(t)\right]$ are functional sets for each $r \in[0,1]$, 
Step (iii): using Equation (8) to construct a solution $x(t)$ such that

$$
[x(t)]^{r}=\left[\underline{x}_{r}(t), \bar{x}_{r}(t)\right], r \in[0,1]
$$

Case II. If $x(t)$ is differentiable, then

$$
\left[D_{2} x(t)\right]^{r}=\left[\bar{x}_{r}^{\prime}(t), \underline{x}_{r}^{\prime}(t)\right]
$$

And to solve (FIVP) we follow the following steps:

Step (i): to solve the system of (ODEs) subsequent for $\underline{x}_{r}(t), \bar{x}_{r}(t)$ :

$$
\begin{aligned}
& \underline{x}_{r}^{\prime}(t)=f_{2, r}\left(t, \underline{x}_{r}(t), \bar{x}_{r}(t)\right) \\
& \bar{x}_{r}^{\prime}(t)=f_{1, r}\left(t, \underline{x}_{r}(t), \bar{x}_{r}(t)\right)
\end{aligned}
$$

Subject to the initial conditions

$$
\begin{aligned}
& \underline{x}_{r}\left(t_{0}\right)=\underline{x}_{r}^{0}(t) \\
& \bar{x}_{r}\left(t_{0}\right)=\bar{x}_{r}^{0}(t)
\end{aligned}
$$

Step (ii): ensure that the solution $\left[\underline{x}_{r}(t), \bar{x}_{r}(t)\right]$ and its derivative $\left[\bar{x}_{r}^{\prime}(t), \underline{x}_{r}^{\prime}(t)\right]$ are functional sets for each $r \in[0,1]$,

Step (iii): using Equation (8) to construct a solution $x(t)$ such that

$$
[x(t)]^{r}=\left[\underline{x}_{r}(t), \bar{x}_{r}(t)\right], r \in[0,1]
$$

Case III. Gather approximate iterations $x_{0}(t), x_{1}(t), \cdots, x_{n}(t)$

$$
\psi_{\underline{x}_{r, k}}(t)=\sum_{m=0}^{k-1} \underline{x}_{r, m}(t) \text { and } \psi_{\bar{x}_{r, k}}(t)=\sum_{m=0}^{k-1} \bar{x}_{r, m}(t)
$$

its value determined by the number of limits after summing the approximate iterations $k$, representing the number of iterations, when collecting the frequencies we substitute for the best values of $\bar{h}, \bar{h}=-1$ so that the sequence (34) is convergent we get the sequenced solution to the problem of elementary fuzzy values in an analytical homotopy method.

Case IIII. Using the Padè approximation $[N / M]$ where $M, N \in \mathbb{N} U\{0\}$ we obtain:

$$
P A[N / M]=\frac{P_{N}(x)}{Q_{M}(x)}=\frac{\sum_{v=0}^{N} b_{v} x^{v}}{1+\sum_{z=1}^{M} c_{z} x^{z}}
$$

By taking different values of $t$ and substituting them into the Padè sequence, (35), we obtain approximate solutions in an analytical homotopy method with the upper and lower Padè approximations.

Example 4.1. Consider the following linear (FIVP) [29]

$$
x_{r}^{\prime}(t)=2 t x(t)+t u, 0 \leq t \leq 1
$$

Subject to the fuzzy initial condition

$$
x(0)=u, \text { where } u=[r-1,1-r], x(0)=[r-1,1-r]
$$

The fuzzy (1)-differentiable exact solution is $\underline{x}(t)=\frac{1}{2}\left(3 \mathrm{e}^{t^{2}}-1\right)(r-1)$. 
When the fuzzy (2), differentiable exact of the solution is $\bar{x}(t)=\frac{1}{2}\left(3 \mathrm{e}^{t^{2}}-1\right)(1-r)$.

\section{Solution:}

The first Case 1: When the system of the (ODEs) matching to (1) and (2), then differentiability is

The first step: If $x(t)$ is differentiable (1) then

$$
\begin{aligned}
& \underline{x}_{r}^{\prime}(t)=2 t \underline{x}_{r}(t)+t(r-1) \\
& \bar{x}_{r}^{\prime}(t)=2 t \bar{x}_{r}(t)+t(1-r)
\end{aligned}
$$

Subject to the initial condition

$$
\begin{aligned}
& \underline{x}_{r}(0)=(r-1) \\
& \bar{x}_{r}(0)=(1-r)
\end{aligned}
$$

The second step:

To find the iterations $\underline{x}_{r, 1}(t), \bar{x}_{r, 1}(t), \cdots$ lower and upper we will use the equation to find the homotopic equation in the formula:

$$
\begin{aligned}
& \underline{x}_{r, m}(t)=X_{m} \underline{x}_{r, m-1}(t)+\bar{h} \int_{t_{0}}^{t} R \underline{x}_{r, m}\left(\underline{\boldsymbol{x}}_{r, m-1}(\tau), \overline{\boldsymbol{x}}_{r, m-1}(\tau)\right)\left(1-x_{m}\right) \mathrm{d} \tau \\
& \bar{x}_{r, m}(t)=X_{m} \bar{x}_{r, m-1}(t)+\bar{h} \int_{t_{0}}^{t} R \bar{x}_{r, m}\left(\underline{\boldsymbol{x}}_{r, m-1}(\tau), \overline{\boldsymbol{x}}_{r, m-1}(\tau)\right)\left(1-x_{m}\right) \mathrm{d} \tau
\end{aligned}
$$

Through the equation for the deformation of order $m,(40) m \geq 1$, we get:

$$
\begin{aligned}
& \underline{x}_{r, m}(t)=X_{m} \underline{x}_{r, m-1}(t)+\bar{h} \int_{t_{0}}^{t} \underline{x}_{r, m-1}^{\prime}(t)-2 \underline{x}_{r, m-1}(t)-t(r-1)\left(1-x_{m}\right) \mathrm{d} \tau \\
& \bar{x}_{r, m}(t)=X_{m} \bar{x}_{r, m-1}(t)+\bar{h} \int_{t_{0}}^{t} \bar{x}_{r, m-1}^{\prime}(t)-2 t \bar{x}_{r, m-1}(t)-t(1-r)\left(1-x_{m}\right) \mathrm{d} \tau
\end{aligned}
$$

When $m=1,2,3,4$ by substituting into Equation (41) we get:

$$
\begin{gathered}
x_{r, 1}(t)=-\frac{3}{2} \bar{h} t^{2} r+\frac{3}{2} \bar{h} t^{2} \\
\bar{x}_{r, 1}(t)=-\frac{3}{2} \bar{h} t^{2}+\frac{3}{2} \bar{h} t^{2} r \\
\underline{x}_{r, 2}(t)=-\frac{3}{2} \bar{h} t^{2} r+\frac{3}{2} \bar{h} t^{2}+\frac{3}{4} \bar{h}^{2} t^{4} r-\frac{3}{4} \bar{h}^{2} t^{4}-\frac{3}{2} \bar{h}^{2} t^{2} r+\frac{3}{2} \bar{h}^{2} t^{2} \\
\bar{x}_{r, 2}(t)=-\frac{3}{2} \bar{h} t^{2}+\frac{3}{2} \bar{h} t^{2} r+\frac{3}{4} \bar{h}^{2} t^{4}-\frac{3}{4} \bar{h}^{2} t^{4} r-\frac{3}{2} \bar{h}^{2} t^{2}+\frac{3}{2} \bar{h}^{2} t^{2} r
\end{gathered}
$$

\section{The third case:}

Sum the approximate iterations lower and upper $\underline{x}_{r, 0}(t), \bar{x}_{r, 0}(t), x_{r, 1}(t), \bar{x}_{r, 1}(t), \cdots$.

After substituting in the best values of, $\bar{h}, \bar{h}=-1, r=0.9$, we get an approximate solution as follows:

$$
\begin{gathered}
\psi_{\underline{x}_{r, k}}(t)=\sum_{m=0}^{k-1} \underline{x}_{r, m}(t), \quad \psi_{\bar{x}_{r, k}}(t)=\sum_{m=0}^{k-1} \bar{x}_{r, m}(t) \\
\psi_{\underline{x}_{r, 5}}(t)=-0.00625 t^{8}-0.02500 t^{6}-0.07500 t^{4}-0.15000 t^{2}-0.1 \\
\psi_{\bar{x}_{r, 5}}(t)=0.00625 t^{8}+0.02500 t^{6}+0.07500 t^{4}+0.15000 t^{2}+0.1
\end{gathered}
$$

Fourth case: We connect the solution series (42) to Padè approximation 
$P A[N / M]$ and we get:

$$
\begin{gathered}
P A[N / M]=\frac{P_{N}(x)}{Q_{M}(x)}=\frac{\sum_{v=0}^{N} b_{v} x^{v}}{1+\sum_{z=1}^{M} c_{z} x^{2}} \\
\text { lower } P A[4 / 4]=\frac{-0.09999-0.09999 t^{2}-0.00833 t^{4}}{0.99999-0.49999 t^{2}+0.08333 t^{4}} \\
\text { upper }: P A[4 / 4]=\frac{0.09999+0.09999 t^{2}+0.00833 t^{4}}{0.99999-0.49999 t^{2}+0.08333 t^{4}}
\end{gathered}
$$

By taking different values of $(t)$ and substituting them into the Padè series (44), we obtain approximate solutions by homotopic analytical method with Padè approximation, as shown in Table 1 .

\section{The second case II:}

\section{The first step:}

For find the upper and lower values of $\underline{x}_{r, m}(t), \bar{x}_{r, m}(t)$, if $x(t)$ is differentiable then:

$$
\begin{aligned}
& \underline{x}_{r}^{\prime}(t)=2 t \bar{x}_{r}(t)+t(r-1) \\
& \bar{x}_{r}^{\prime}(t)=2 \underline{x}_{r}(t)+t(1-r)
\end{aligned}
$$

subject to the initial condition

$$
\begin{aligned}
& \underline{x}_{r}(0)=(r-1) \\
& \bar{x}_{r}(0)=(1-r)
\end{aligned}
$$

\section{The second step:}

To find the iterations $x_{r, 1}(t), x_{r, 2}(t), x_{r, 3}(t), \cdots$ upper and lower by using the equations

$$
\begin{aligned}
& \underline{x}_{r, m}(t)=X_{m} \bar{x}_{r, m-1}(t)+\bar{h} \int_{t_{0}}^{t} R \bar{x}_{r, m}\left(\underline{x}_{r, m-1}(\tau), \overline{\boldsymbol{x}}_{r, m-1}(\tau)\right)\left(1-x_{m}\right) \mathrm{d} \tau \\
& \bar{x}_{r, m}(t)=X_{m} \underline{x}_{r, m-1}(t)+\bar{h} \int_{t_{0}}^{t} R \underline{x}_{r, m}\left(\underline{\boldsymbol{x}}_{r, m-1}(\tau), \overline{\boldsymbol{x}}_{r, m-1}(\tau)\right)\left(1-x_{m}\right) \mathrm{d} \tau
\end{aligned}
$$

When $m=1$, by substituting in the upper and lower Equations (47) we get:

$$
\begin{aligned}
& x_{r, 1}(t)=-\frac{3}{2} \bar{h} t^{2} r+\frac{3}{2} \bar{h} t^{2} \\
& \bar{x}_{r, 1}(t)=-\frac{3}{2} \bar{h} t^{2}+\frac{3}{2} \bar{h} t^{2} r
\end{aligned}
$$

When $m=2,3, \cdots$ in Equation (54) and using the Riemann-Liouville integral properties, we get:

$$
\begin{aligned}
& \underline{x}_{r, 2}(t)=-\frac{3}{2} \bar{h} t^{2} r+\frac{3}{2} \bar{h} t^{2}+\frac{3}{4} \bar{h}^{2} t^{4} r-\frac{3}{4} \bar{h}^{2} t^{4}-\frac{3}{2} \bar{h}^{2} t^{2} r+\frac{3}{2} \bar{h}^{2} t^{2} \\
& \bar{x}_{r, 2}(t)=-\frac{3}{2} \bar{h} t^{2}+\frac{3}{2} \bar{h} t^{2} r+\frac{3}{4} \bar{h}^{2} t^{4}-\frac{3}{4} \bar{h}^{2} t^{4} r-\frac{3}{2} \bar{h}^{2} t^{2}+\frac{3}{2} \bar{h}^{2} t^{2} r
\end{aligned}
$$

\section{The third case III:}

We assume the upper and lower approximate iterations $x_{r, 0}(t), x_{r, 1}(t) x_{r, 2}(t), x_{r, 3}(t), x_{r, 4}(t)$.

After substituting in the best values of $\bar{h}, \bar{h}=-1$, we get an approximate 
solution of the upper and lower values

$$
\begin{aligned}
& \psi_{x_{r, 5}}(t)=-0.00625 t^{8}-0.02500 t^{6}-0.07500 t^{4}-0.15000 t^{2}-0.1 \\
& \psi_{\bar{x}_{r, 5}}(t)=0.00625 t^{8}+0.02500 t^{6}+0.07500 t^{4}+0.15000 t^{2}+0.1
\end{aligned}
$$

\section{The fourth step:}

We connect the solution series (48) to the approximations of the upper and lower pads $P A[N / M]$ and we get:

$$
P A[N / M]=\frac{P_{N}(x)}{Q_{M}(x)}=\frac{\sum_{v=0}^{N} b_{v} x^{v}}{1+\sum_{z=1}^{M} c_{z} x^{z}}
$$

By taking different values of $(t)$ and substituting them into the Padè series (49), we get approximate solutions in an analytical homotopic method with upper and lower Padè approximations as shown in Table 1, Table 2, and Figure 1, Figure 2.

Example 4.2. Consider the following linear (FIVP) [30]

$$
y_{r}^{\prime}(t)=y(t), 0 \leq t \leq 1
$$

Subject to the fuzzy initial condition

$$
\underline{y}(0)=(r-1), \bar{y}(0)=(1-r)
$$

The fuzzy (1)-differentiable exact solution is $\underline{y}(t)=(r-1) \mathrm{e}^{t}$.

When the fuzzy (2)-differentiable exact of the solution is $\bar{y}(t)=(1-r) \mathrm{e}^{t}$.

Solution: The first Case 1: When the system of the (ODEs) equivalent to (1) and (2), then differentiability is

The first step: If $y(t)$ is differentiable (1) and (2) then:

Table 1. Lower numerical results for both the exact solution and homotopic approximation and homotopic approximation-Padè approximation PA [4/4] at different values of $(t)$ for example (4.1).

\begin{tabular}{cccccc}
\hline$t$ & Exact Solution & Approximation & Absolute Error & Approximation & Absolute Error \\
& & PAD $\underline{x}$ & $\underline{x}$ & \\
\hline 0 & -0.10 & -0.10 & 0 & -0.10 & 0 \\
0.1 & -0.10150 & -0.10150 & $1.25208 \times 10^{-13}$ & -0.10150 & $2.10428 \times 10^{-14}$ \\
0.2 & -0.10612 & -0.10612 & $1.28858 \times 10^{-10}$ & -0.10612 & $2.22060 \times 10^{-11}$ \\
0.3 & -0.11412 & -0.11412 & $7.49328 \times 10^{-9}$ & -0.11412 & $1.34668 \times 10^{-9}$ \\
0.4 & -0.12602 & -0.12602 & $1.34648 \times 10^{-7}$ & -0.12602 & $2.56747 \times 10^{-8}$ \\
0.5 & -0.14260 & -0.14260 & $1.27344 \times 10^{-6}$ & -0.14260 & $2.62207 \times 10^{-7}$ \\
0.6 & -0.16499 & -0.16499 & $8.03618 \times 10^{-6}$ & -0.16499 & $1.81950 \times 10^{-6}$ \\
0.7 & -0.16499 & -0.19480 & $3.84079 \times 10^{-5}$ & -0.19483 & $9.74307 \times 10^{-6}$ \\
0.8 & -0.23447 & -0.23432 & $1.49955 \times 10^{-4}$ & -0.23442 & $4.34537 \times 10^{-5}$ \\
0.9 & -0.28718 & -0.28668 & $5.02252 \times 10^{-4}$ & -0.28701 & $1.69612 \times 10^{-4}$ \\
1.0 & -0.35774 & -0.35625 & $1.49227 \times 10^{-3}$ & -0.35714 & $5.99417 \times 10^{-4}$ \\
\hline
\end{tabular}


Table 2. Upper numerical results for both the exact solution and homotopy approximation and homotopy approximation-Padè approximation $P A[4 / 4]$ at different values of $(t)$ for example (4.1).

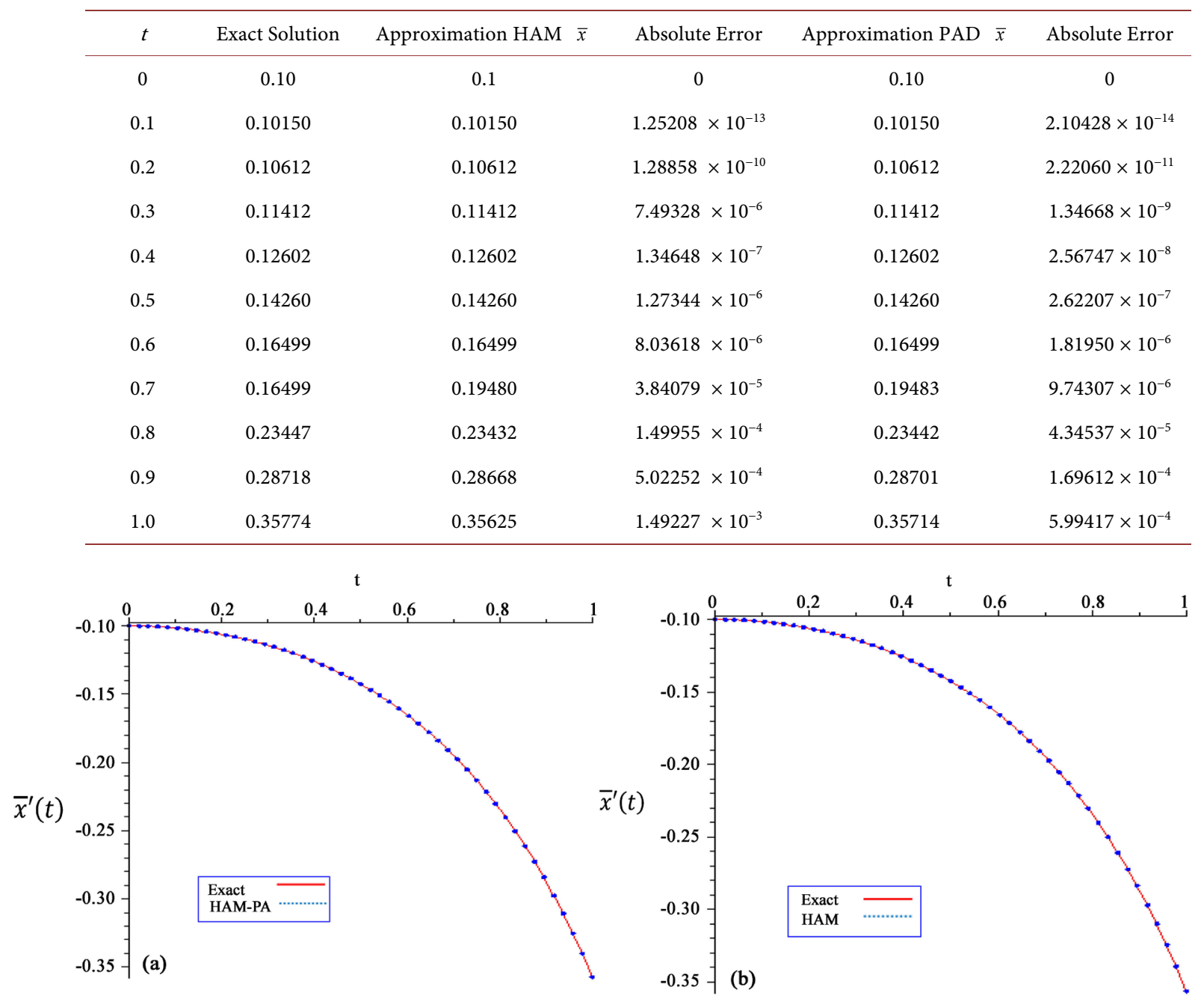

Figure 1. An illustrative comparison between an exact solution with both the analytical lower homotopy method and the analytical homotopy method-Padè approximation when $m=5$ for example (4.1). (a) Exact solution with homotopy analytical method-Padè approximation; (b) Exact solution with homotopy analytical method.
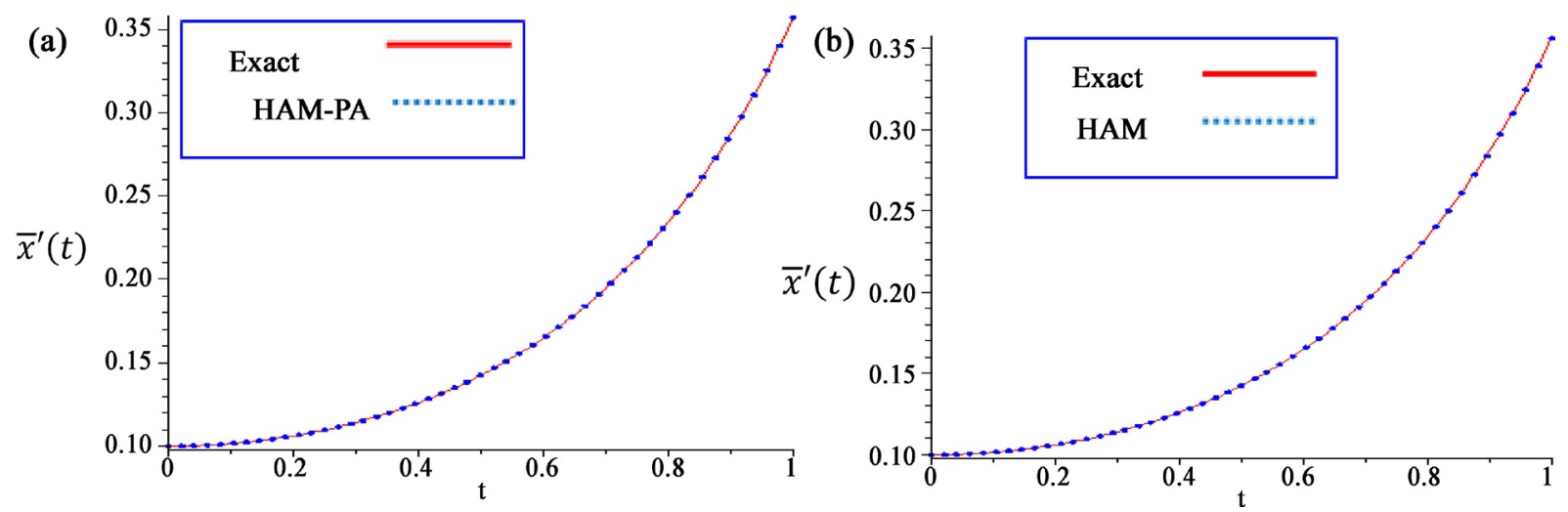

Figure 2. An illustrative comparison between an exact solution with both the analytical upper homotopy method and the analytical homotopy method-Padè approximation when $m=5$ for example (4.1). (a) Exact solution with homotopy analytical method-Padè approximation; (b) Exact solution with homotopy analytical method. 


$$
\begin{aligned}
& \underline{y}_{r}^{\prime}(t)=\underline{y}_{r}(t) \\
& \bar{y}_{r}^{\prime}(t)=\bar{y}_{r}(t)
\end{aligned}
$$

subject to the initial condition

$$
\begin{aligned}
& \underline{y}_{r}(0)=(r-1) \\
& \bar{y}_{r}(0)=(1-r)
\end{aligned}
$$

The second step: To find the iterations $\underline{y}_{r, 1}(t), \bar{y}_{r, 1}(t), \cdots$ lower and upper we will use the equation to find the homotopic equation:

$$
\begin{aligned}
& \underline{y}_{r, m}(t)=X_{m} \underline{y}_{r, m-1}(t)+\bar{h} \int_{t_{0}}^{t} R \underline{y}_{r, m}\left(\underline{\boldsymbol{y}}_{r, m-1}(\tau), \overline{\boldsymbol{y}}_{r, m-1}(\tau)\right)\left(1-x_{m}\right) \mathrm{d} \tau \\
& \bar{y}_{r, m}(t)=x_{m} \bar{y}_{r, m-1}(t)+\bar{h} \int_{t_{0}}^{t} R \bar{y}_{r, m}\left(\underline{\boldsymbol{y}}_{r, m-1}(\tau), \overline{\boldsymbol{y}}_{r, m-1}(\tau)\right)\left(1-x_{m}\right) \mathrm{d} \tau
\end{aligned}
$$

When $m=1,2,3,4$ by substituting into the Equation (54), we get:

$$
\begin{gathered}
\underline{y}_{r, 1}(t)=\bar{h} t-\bar{h} r t, \quad \bar{y}_{r, 1}(t)=-\bar{h} t+\bar{h} r t \\
\underline{y}_{r, 2}(t)=\bar{h} t-\bar{h} r t-\frac{1}{2} \bar{h}^{2} t^{2}+\frac{1}{2} \bar{h}^{2} t^{2} r+\bar{h}^{2} t-\bar{h}^{2} r t \\
\bar{y}_{r, 2}(t)=-\bar{h} t+\bar{h} r t+\frac{1}{2} \bar{h}^{2} t^{2}-\frac{1}{2} \bar{h}^{2} t^{2} r-\bar{h}^{2} t+\bar{h}^{2} r t
\end{gathered}
$$

The third case: Sum the approximate iterations lower and upper $y_{r, 0}(t), y_{r, 1}(t), \cdots$.

After substituting in the best values of $\bar{h}, \bar{h}=-1, r=0.9$, we get an approximate solution as follows:

$$
\begin{gathered}
\psi_{\underline{y}_{r, k}}(t)=\sum_{m=0}^{k-1} \underline{y}_{r, m}, \psi_{\bar{y}_{r, k}}(t)=\sum_{m=0}^{k-1} \bar{y}_{r, m} \\
\psi_{\underline{y}_{r, 5}}(t)=-0.00416 t^{4}-0.01666 t^{3}-0.05000 t^{2}-0.1 t-0.1 \\
\psi_{\bar{y}_{r, 5}}(t)=0.00416 t^{4}+0.01666 t^{3}+0.05000 t^{2}+0.1 t+0.1
\end{gathered}
$$

Fourth case: We connect the solution series (55) to Padè approximation $P A[N / M]$ and we get:

$$
\begin{gathered}
P A[N / M]=\frac{P_{N}(x)}{Q_{M}(x)}=\frac{\sum_{v=0}^{N} b_{v} x^{v}}{1+\sum_{z=1}^{M} c_{z} x^{2}} \\
\text { lower }: P A[1 / 3]=\frac{-0.1-0.25000 t}{1.0-0.74999 t+0.24999 t^{2}-0.04166 t^{3}} \\
\text { upper : } P A[1 / 3]=\frac{0.1+0.02500 t}{1.0-0.74999 t+0.24999 t^{2}-0.04166 t^{3}}
\end{gathered}
$$

By taking different values of $(t)$ and substituting them into the Padè series (57), we obtain approximate solutions by homotopic analytical method with Padè approximation, as shown in Table 3.

The second case II: The first step:

For find the upper and lower values of $\bar{x}_{r, m}(t), \underline{x}_{r, m}(t)$, if $x(t)$ is differentiable then:

$$
\begin{aligned}
& \underline{y}_{r}^{\prime}(t)=\underline{y}_{r}(t) \\
& \bar{y}_{r}^{\prime}(t)=\bar{y}_{r}(t)
\end{aligned}
$$


Subject to the initial condition

$$
\begin{aligned}
& \underline{y}_{r}(0)=(r-1) \\
& \bar{y}_{r}(0)=(1-r)
\end{aligned}
$$

The second step: To find the iterations $y_{r, 1}(t), y_{r, 2}(t), \cdots$ upper and lower by using the equations

$$
\begin{aligned}
& \underline{y}_{r, m}(t)=X_{m} \bar{y}_{r, m-1}(t)+\bar{h} \int_{t_{0}}^{t} R \bar{y}_{r, m}\left(\underline{\boldsymbol{y}}_{r, m-1}(\tau), \overline{\boldsymbol{y}}_{r, m-1}(\tau)\right)\left(1-x_{m}\right) \mathrm{d} \tau \\
& \bar{y}_{r, m}(t)=X_{m} \underline{y}_{r, m-1}(t)+\bar{h} \int_{t_{0}}^{t} R \underline{y}_{r, m}\left(\underline{\boldsymbol{y}}_{r, m-1}(\tau), \overline{\boldsymbol{y}}_{r, m-1}(\tau)\right)\left(1-x_{m}\right) \mathrm{d} \tau
\end{aligned}
$$

The third case: We assume the upper and lower approximate iterations $y_{r, 0}(t), y_{r, 1}(t), \cdots$.

After substituting in the best values of $\bar{h}, \bar{h}=-1$, we get an approximate solution of the upper and lower values

$$
\begin{aligned}
& \psi_{\underline{y}_{r, 5}}(t)=-0.00416 t^{4}-0.01666 t^{3}-0.05000 t^{2}-0.1 t-0.1 \\
& \psi_{\bar{y}_{r, 5}}(t)=0.00416 t^{4}+0.01666 t^{3}+0.05000 t^{2}+0.1 t+0.1
\end{aligned}
$$

\section{The fourth step:}

We connect the solution series (61) to the approximations of the upper and lower pads $P A[N / M]$ and we get:

$$
P A[N / M]=\frac{P_{N}(x)}{Q_{M}(x)}=\frac{\sum_{v=0}^{N} b_{v} x^{v}}{1+\sum_{z=1}^{M} c_{z} x^{z}}
$$

By taking different values of $(t)$ and substituting them into the Padè series (62), we get approximate solutions in an analytical homotopy method with upper and lower Padè approximations as shown in Table 3, Table 4, and Figure 3, Figure 4.

Table 3. Lower numerical results for both the exact solution and homotopy approximation and homotopy approximation-Padè approximation $P A[1 / 3]$ at different values of ( $t$ ) for example (4.2).

\begin{tabular}{cccccc}
\hline$t$ & Exact Solution & $\begin{array}{c}\text { Approximation } \\
\text { HAM } \underline{x}\end{array}$ & Absolute Error & $\begin{array}{c}\text { Approximation } \\
\text { PAD } \underline{x}\end{array}$ & Absolute Error \\
\hline 0 & -0.1 & -0.1 & 0 & -0.1 & 0 \\
0.1 & 0.11051 & 0.11051 & $8.47423 \times 10^{-9}$ & -0.11051 & $2.40151 \times 10^{-9}$ \\
0.2 & -0.12214 & -0.12214 & $2.75816 \times 10^{-7}$ & -0.12214 & $8.86663 \times 10^{-8}$ \\
0.3 & 0.13498 & -0.13498 & $2.13075 \times 10^{-6}$ & -0.13498 & $7.77537 \times 10^{-7}$ \\
0.4 & -0.14918 & -0.14917 & $9.13643 \times 10^{-6}$ & -0.14918 & $3.78701 \times 10^{-6}$ \\
0.5 & -0.16487 & -0.16484 & $2.83770 \times 10^{-5}$ & -0.16488 & $1.33691 \times 10^{-5}$ \\
0.6 & -0.18221 & -0.18214 & $7.18800 \times 10^{-5}$ & -0.18225 & $3.85161 \times 10^{-5}$ \\
0.7 & -0.20137 & -0.20121 & $1.58187 \times 10^{-4}$ & -0.20147 & $9.64731 \times 10^{-5}$ \\
0.8 & -0.22255 & -0.22224 & $3.14092 \times 10^{-4}$ & -0.22277 & $2.18184 \times 10^{-4}$ \\
0.9 & -0.24596 & -0.24538 & $5.76561 \times 10^{-4}$ & -0.24641 & $4.56586 \times 10^{-4}$ \\
1.0 & -0.27182 & -0.27083 & $9.94849 \times 10^{-4}$ & -0.27272 & $8.99089 \times 10^{-4}$ \\
\hline
\end{tabular}


Table 4. Upper numerical results for both the exact solution and homotopic approximation and homotopy approximation-Padè approximation $P A[1 / 3]$ at different values of $(t)$ for example (4.2).

\begin{tabular}{|c|c|c|c|c|c|}
\hline$t$ & Exact Solution & Approximation HAM $\bar{x}$ & Absolute Error & Approximation PAD $\bar{x}$ & Absolute Error \\
\hline 0 & 0.1 & 0.1 & 0 & 0.1 & 0 \\
\hline 0.1 & 0.11051 & 0.11051 & $8.47423 \times 10^{-9}$ & 0.11051 & $2.40151 \times 10^{-9}$ \\
\hline 0.2 & 0.12214 & 0.12214 & $2.75816 \times 10^{-7}$ & 0.12214 & $8.86663 \times 10^{-8}$ \\
\hline 0.3 & 0.13498 & 0.13498 & $2.13075 \times 10^{-6}$ & 0.13498 & $7.77537 \times 10^{-7}$ \\
\hline 0.4 & 0.14918 & 0.14917 & $9.13643 \times 10^{-3}$ & 0.14918 & $3.78701 \times 10^{-6}$ \\
\hline 0.5 & 0.16487 & 0.16484 & $2.83770 \times 10^{-5}$ & 0.16488 & $1.33691 \times 10^{-5}$ \\
\hline 0.6 & 0.18221 & 0.18214 & $7.18800 \times 10^{-5}$ & 0.18225 & $3.85161 \times 10^{-5}$ \\
\hline 0.7 & 0.20137 & 0.20121 & $1.58187 \times 10^{-4}$ & 0.20147 & $9.64731 \times 10^{-5}$ \\
\hline 0.8 & 0.22255 & 0.22224 & $3.14092 \times 10^{-4}$ & 0.22277 & $2.18184 \times 10^{-4}$ \\
\hline 0.9 & 0.24596 & 0.24538 & $5.76561 \times 10^{-4}$ & 0.24641 & $4.56586 \times 10^{-4}$ \\
\hline 1.0 & 0.27182 & 0.27083 & $9.94849 \times 10^{-4}$ & 0.27272 & $8.99089 \times 10^{-4}$ \\
\hline
\end{tabular}
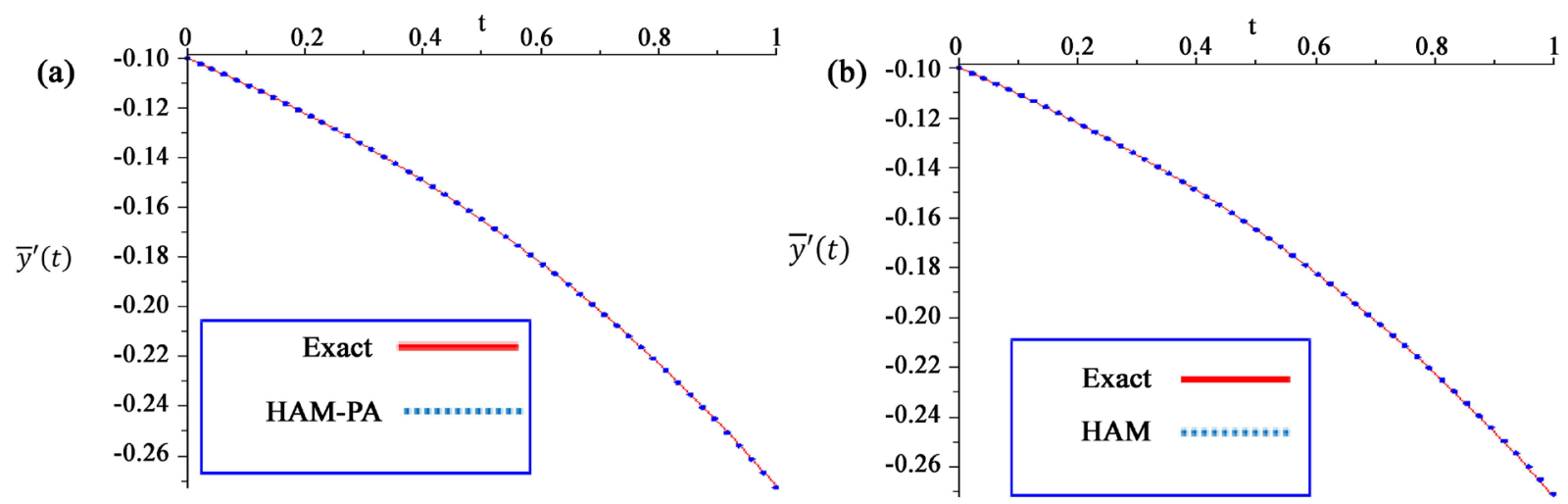

Figure 3. An illustrative comparison between an exact solution with both the analytical lower homotopy method and the analytical homotopy method-Pade approximation when $\mathrm{m}=5$ for example (4.2). (a) Exact solution with homotopy analytical method-Padè approximation; (b) Exact solution with homotopy analytical method.
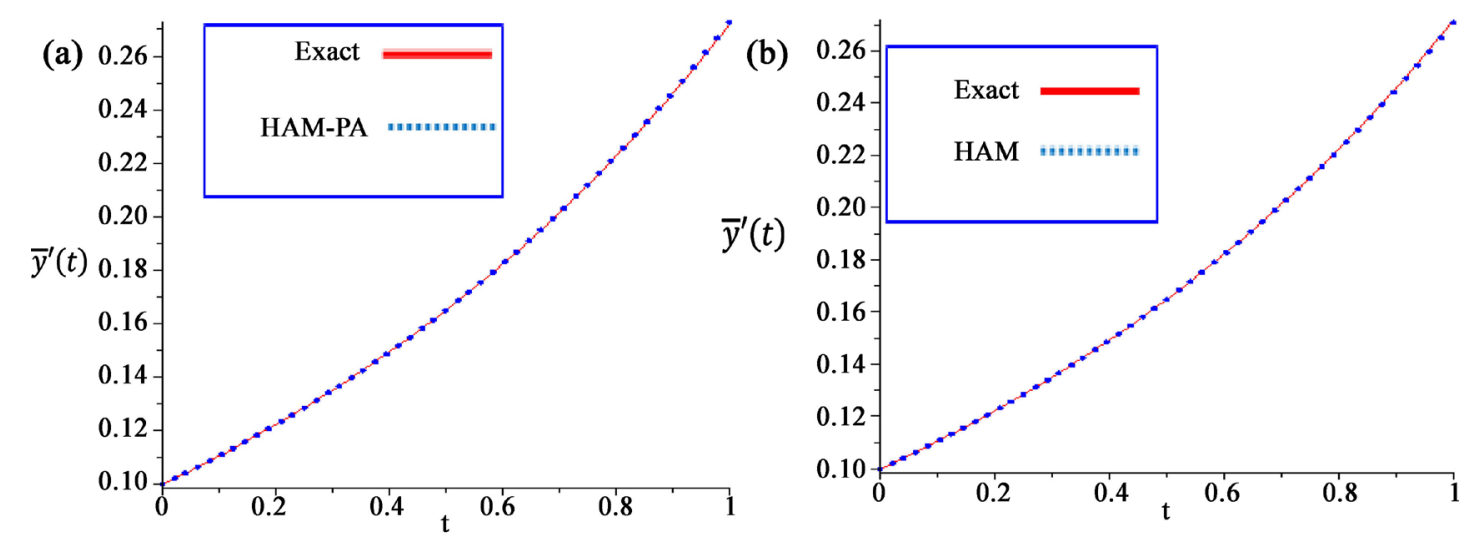

Figure 4. An illustrative comparison between an exact solution with both the analytical upper homotopy method and the analytical homotopy method-Padè approximation when $m=5$ for example (4.2). (a) Exact solution with homotopy analytical method Padè approximation; (b) Exact solution with homotopy analytical method. 


\section{Conclusion}

In the case of fuzzy differential equations, it is found that the results of numerical solutions of the homotopy method with Padè approximations are very close when compared with the exact solutions (accurate). There is no problem with this sentence and it has been validated. The proposed method is very powerful, straightforward, and promising algorithm in finding a solution to the analytical approximate form of broad categories of Fuzzy Initial Values Problems (FIVPS).

\section{Acknowledgements}

For the University of Al-Mosul College of Education for Pure Sciences Department of Mathematics for the assistance they provided to improve the quality of this work.

\section{Conflicts of Interest}

The authors declare no conflicts of interest regarding the publication of this paper.

\section{References}

[1] He, J. (1999) Homotopy Perturbation Technique. Computer Methods in Applied Mechanics and Engineering, 178, 257-262. https://doi.org/10.1016/S0045-7825(99)00018-3

[2] Zadeh, L.A. (1965) Fuzzy Sets. Information and Control, 8, 338-353. https://doi.org/10.1016/S0019-9958(65)90241-X

[3] Zadeh, L.A. (1978) Fuzzy Sets as a Basis for a Theory of Possibility. Fuzzy Sets and Systems, 1, 3-28. https://doi.org/10.1016/0165-0114(78)90029-5

[4] Datta, D.P. (2003) The Golden Mean, Scale Free Extension of Real Number System, Fuzzy Sets and (1/f) Spectrum in Physics and Biology. Chaos, Solitons and Fractals, 17, 781-788. https://doi.org/10.1016/S0960-0779(02)00531-3

[5] Zurigat, M., Momani, S., Odibat, Z. and Alawneh, A. (2010) The Homotopy Analytical Method for Handling Systems of Fractional Differential Equations. Applied Mathematical Modelling, 34, 24-35. https://doi.org/10.1016/j.apm.2009.03.024

[6] Hanss, M. (2005) Applied Fuzzy Arithmetic: An Introduction with Engineering Applications. Springer-Verlag, Berlin.

[7] Guo, M., Xue, X. and Li, R. (2003) Impulsive Functional Differential Inclusions and Fuzzy Population Models. Fuzzy Sets and Systems, 138, 601-615. https://doi.org/10.1016/S0165-0114(02)00522-5

[8] El Naschie, M.S. (2005) On a Fuzzy Kahler-Like Manifold Which Is Consistent with the Two Slit Experiment. International Journal of Nonlinear Sciences and Numerical Simulation, 6, 95-98. https://doi.org/10.1515/IJNSNS.2005.6.2.95

[9] Barro, S. and Marin, R. (2002) Fuzzy Logic in Medicine. Physica-Verlag, Heidelberg. https://doi.org/10.1007/978-3-7908-1804-8

[10] Liao, S.J. and Cheung, K.F. (2003) Homotopy Analysis Method of Nonlinear Progressive Waves in Deep Water. Journal of Engineering Mathematics, 45, 105-116. https://doi.org/10.1023/A:1022189509293

[11] Oberguggenberger, M. and Pittschmann, S. (1999) Differential Equations with 
Fuzzy Parameters, Mathematical and Computer Moduling of Dynamical Systems Methods. Tools and Applications in Related Sciences, 5, 181-202. https://doi.org/10.1076/mcmd.5.3.181.3683

[12] Sun, Q. (2005) Solving the Klein-Gordon Equation by Means of the Homotopy Analysis Method. Applied Mathematics and Computation, 169, 355-365.

https://doi.org/10.1016/j.amc.2004.09.056

[13] Wu, W. and Liao, S.J. (2005) Solving Solitary Waves with Discontinuity by Means of the Homotopy Analysis Method. Chaos, Solitons and Fractals, 26, 177-185. https://doi.org/10.1016/j.chaos.2004.12.016

[14] Ahmad, N., Mamat, M., Kavikumar, J. and Hamzah, N. (2012) Solving Fuzzy Duffing's Equation by the Laplace Transform Decomposition. Applied Mathematical Sciences, 6, 2935-2944.

[15] Bencsik, A., Bede, B., Tar, J. and Fodor, J. (2006) Fuzzy Differential Equations in Modeling Hydraulic Differential Servo Cylinders. Third Romanian-Hungarian Joint Symposium on Applied Computational Intelligence (SACD), Timisoara, 1-12.

[16] Liao, S.J. (2006) Series Solutions of Unsteady Boundary-Layer Flows over a Stretching Flat Plate. Studies in Applied Mathematics, 117, 239-263.

https://doi.org/10.1111/j.1467-9590.2006.00354.x

[17] El-Ajou, A., Abu Arqub, O. and Momani, S. (2012) Homotopy Analytical Method for Second-Order Boundary Value Issues of Integro-Differential Equations. Discrete Dynamic in Nature and Society, 2012, Article ID: 365792.

https://doi.org/10.1155/2012/365792

[18] Sharma, P.K. and Chhama, S. (2013) Effect of MHD and Thermal Diffusion on Natural Convection Oscillatory Flow Past Plate. Mathematical Science Letters: An International Journal, 2, 79-86. https://doi.org/10.12785/msl/020202

[19] Abu Arqub, O. and El-Ajou, A. (2013) Solution of the Fractional Epidemic Model by Homotopy Analysis Method. Journal of King Saud University (Science), 25, 73-81. https://doi.org/10.1016/j.jksus.2012.01.003

[20] Hullermeier, E. (1997) An Approach to Modelling and Simulation of Uncertain Systems. International Journal of Uncertainty Fuzziness Knowledge-Based System, 5, 117-137. https://doi.org/10.1142/S0218488597000117

[21] Heba, Sh. and Kais, I. (2018) Solve the Initial Value Problems of Fractional Order Using the Homotopy Analytical Method and Improve Them Using Pade' Approximations. Journal of Education and Science, 28, 142-157. https://doi.org/10.33899/edusj.1999.163332

[22] Liao, S.J. (2003) Beyond Perturbation: Introduction to the Homotopy Analysis Methods. Chapman and Hall/CRC Press, Boca Raton.

[23] Kaleva, O. (1987) Fuzzy Differential Equations. Fuzzy Sets and Systems, 24, 301-317. https://doi.org/10.1016/0165-0114(87)90029-7

[24] Seikkala, S. (1987) On the Fuzzy Initial Value Problem. Fuzzy Sets and Systems, 24, 319-330. https://doi.org/10.1016/0165-0114(87)90030-3

[25] Puri, M.L. and Ralescu, D.A. (1983) Differentials of Fuzzy Functions. Journal of Mathematical Analysis and Applications, 91, 552-558.

https://doi.org/10.1016/0022-247X(83)90169-5

[26] Bede, B. and Gal, S.G. (2005) Generalizations of the Differentiability of Fuzzy Number Value Functions with Applications to Fuzzy Differential Equations. Fuzzy Sets and Systems, 151, 581-599. https://doi.org/10.1016/j.fss.2004.08.001 
[27] Ogunlaran, O.M. and Sagay-Yusuf, H. (2016) Adomain Sumudu Transform Method for the Blasius Equation. British Journal of Mathematics \& Computer Science, 14, 1-8. https://doi.org/10.9734/BJMCS/2016/23104

[28] Al-Hayani, W., Alzubaidy, L. and Entesar, A. (2017) Solutions of Singular of Lane-Emden Type by Homotopy Analysis Method with Genetic Algorithm. Applied Mathematics, 11, 407-416. https://doi.org/10.18576/amis/110208

[29] Omar, A., Ahmad, E., Shaher, M. and Nabil, S. (2013) Analytical Solutions of Fuzzy Initial Value Problems by HAM. Applied Mathematics \& Information Science, 7, 1903-1919. https://doi.org/10.12785/amis/070528

[30] Darabi, P., Moloudzadeh, S. and Khandani, H. (2015) A Numerical Method for Solving First-Order Fully Fuzzy Differential Equation under Strongly Generalized H-Differentiability. Soft Computing, 20, 4085-4098.

https://doi.org/10.1007/s00500-015-1743-0 